\title{
Early response of bone metastases can predict tumor response in patients with non-small-cell lung cancer with bone metastases in the treatment with nivolumab
}

\author{
EIJI NAKATA ${ }^{1}$, SHINSUKE SUGIHARA $^{2}$, YOSHIFUMI SUGAWARA ${ }^{3}$, TOSHIYUKI KOZUKI $^{4}$, \\ DAIJIRO HARADA ${ }^{4}$, NAOYUKI NOGAMI ${ }^{4}$, RYUICHI NAKAHARA ${ }^{1}$, TAKAYUKI FURUMATSU ${ }^{1}$, \\ TOMONORI TETSUNAGA $^{1}$, TOSHIYUKI KUNISADA ${ }^{1}$ and TOSHIFUMI OZAKI ${ }^{1}$
}

\author{
${ }^{1}$ Department of Orthopedic Surgery, Okayama University Hospital, Okayama 700-8558; Departments of ${ }^{2}$ Orthopedic Surgery, \\ ${ }^{3}$ Radiology and ${ }^{4}$ Thoracic Oncology and Medicine, Shikoku Cancer Center, Matsuyama, Ehime 791-0280, Japan
}

Received November 11, 2019; Accepted March 2, 2020

DOI: 10.3892/ol.2020.11856

\begin{abstract}
The effect of nivolumab and the relation between bone response and tumor control in patients with non-small-cell lung cancer (NSCLC) with bone metastases are not clear. The outcome of nivolumab monotherapy was investigated, and whether the response of bone metastases is useful as an early predictor of tumor control in patients with NSCLC with bone metastases was examined. The participants included 15 patients who received nivolumab monotherapy for NSCLC with bone metastases in our institution between 2015 and 2017. Tumor control was defined using Response Evaluation Criteria in Solid Tumors, version 1.1 (RECIST1.1). Response of bone metastases was assessed by the MD Anderson response criteria (MDA criteria). Responses according to RECIST1.1 and the MDA criteria were classified as responder (complete response or partial response) and non-responder [progressive disease (PD) or stable disease]. Progression-free survival (PFS) was investigated using the Kaplan-Meier method. With RECIST1.1, the overall response rate was $20 \%$. Multivariate analysis showed that the MDA criteria were the only risk factor for patients with PD (RECIST1.1). Median PFS was 1.9 months, with PFS of $20 \%$ at 6 months. Univariate analysis showed that being a non-responder according to the MDA criteria was the only risk factor for PFS. In patients who were responders (MDA criteria) within 3 months, PFS was 83 and $50 \%$ at 3 and 6 months, respectively, though all non-responder (MDA criteria) patients converted to PD (RECIST1.1) within 3 months. Response according to RECIST1.1 was significantly correlated with response according to the MDA criteria
\end{abstract}

Correspondence to: Dr Eiji Nakata, Department of Orthopedic Surgery, Okayama University Hospital, 2-5-1 Shikata-cho, Okayama 700-8558, Japan

E-mail: eijinakata8522@yahoo.co.jp

Key words: bone metastasis, non-small-cell lung cancer, programmed cell death 1, nivolumab, Response Evaluation Criteria in Solid Tumors, version 1.1, MD Anderson response criteria
$(\mathrm{P}<0.05)$. In patients who were both responders according to RECIST1.1 and the MDA criteria, time to response with the MDA criteria (1.4-2.0 months) was earlier than with RECIST1.1 (2.8-3.0 months) in all patients. In conclusion, application of the MDA criteria within 2 months of nivolumab monotherapy is useful for early prediction of response and prognosis in patients with NSCLC with bone metastases.

\section{Introduction}

Recently, immune checkpoint inhibitors (ICIs) have been used for the treatment of various malignancies, including non-small-cell lung cancer (NSCLC), renal cell cancer, melanoma, and other cancers (1-4).

Programmed cell death 1 (PD-1) is a receptor expressed on activated $\mathrm{T}$ cells. It binds to its ligands, programmed death-ligand 1 (PD-L1) and programmed death-ligand 2 (PD-L2), expressed in cancer cells, leading to the inhibition of T cell activation $(3,4)$. The interaction of PD-1 with PD-L1 promotes tumor immune escape, which leads to patients with the disease having a poor prognosis. Nivolumab, a fully human IgG4 PD-1 ICI antibody, disrupts PD-1-mediated signaling and restores antitumor immunity $(3,4)$.

In phase II and III studies, nivolumab monotherapy showed good tolerance and excellent clinical efficacy in patients with advanced or recurrent NSCLC, which had progressed after platinum-containing chemotherapy (5-9). In these studies, nivolumab was associated with an overall response rate (ORR) of $15-26 \%$, overall survival (OS) of $41-71 \%$ at 1 year, and progression-free survival (PFS) of $19-25 \%$ at 1 year post-treatment (5-9).

Bone is one of the common metastatic sites of advanced lung cancer. Approximately 30-66\% of patients with advanced NSCLC develop bone metastases during the course of their disease $(10,11)$.

Bone metastases usually present as lytic, blastic, or mixed lesions.Lytic or mixed metastatic lesions that have been successfully treated show osteosclerotic change (OC) on computed tomography $(\mathrm{CT})$ due to the reparative process known as re-ossification (12-14). OC has been reported as an indicator of 
good therapeutic response to treatment with various anticancer therapies, such as endocrine therapy, molecular targeted therapy, bone-modifying agents (BMAs), radiotherapy (RT), and a combination of these treatments (12-21). Yamashita et al reported $\mathrm{OC}$ as being an indicator of a good therapeutic response in lung cancer patients with bone metastases treated with gefitinib (15). The authors showed that the OC group had a significantly higher ORR and improved OS compared to the no OC (NOC) group. Rong et al reported a median interval of 2 months when OC occurred following chemotherapy in lung cancer patients with bone metastases (16). The authors showed that the OC group had significantly higher 3-month disease control rate (DCR) and 1-year PFS than the NOC group. However, these reports are inadequate, because they do not take into account the regression of extraskeletal lesions, which can also be a predictor of treatment as defined in the Response Evaluation Criteria in Solid Tumors, version 1.1 (RECIST1.1).

In 2004, Hamaoka established the MD Anderson response classification criteria (MDA criteria), specific for the assessment of bone metastases (12-14,21). The MDA criteria divide the responses into 4 categories and can assess both the regression of extraskeletal lesions and OC. The MDA criteria allow a greater range of bone lesions to be considered measurable disease than RECIST1.1 by allowing measurement of numerous types of bone lesions regardless of soft tissue extension (12-14). Since their introduction, several authors reported their usefulness for the assessment of therapeutic response to chemotherapy and RT in patients with bone metastases (12-14,21). Hamaoka reported that the MDA classification is superior to the WHO classification in differentiating between the responders and non-responders in breast cancer patients with bone-only metastases (12). According to the MDA criteria, there were significant differences in PFS between patients classified as responders and those classified as non-responders $(\mathrm{P}=0.025)$, but not with the WHO criteria. We previously reported that the MDA criteria were useful for assessment of radiological responses of irradiated vertebrae (21). There was a significant trend that, with a better response assessed by the MDA criteria, there were more patients without pain $(\mathrm{P}=0.021)$.

Although early prediction of the effects of chemotherapy for NSCLC patients would help guide clinical practice, the optimal markers for predicting the outcomes in NSCLC patients are unknown, particularly in patients with bone metastases after ICI treatment. To the best of our knowledge, no studies have focused on the effect of nivolumab in NSCLC patients with bone metastases. Moreover, no studies have investigated the relationship between bone response and tumor control with nivolumab treatment.

Therefore, the clinical outcome of nivolumab monotherapy was investigated in NSCLC patients with bone metastases. Furthermore, whether the response of bone metastases assessed by the MDA criteria can be used as an early imaging predictor of tumor control in NSCLC patients with bone metastases was explored.

\section{Patients and methods}

Study population. The records of 52 patients who received nivolumab monotherapy for stage IV or recurrent NSCLC in our institution between December 2015 (the date nivolumab was approved in Japan) and March 2017 were retrospectively reviewed. The last follow-up evaluation of patients was performed in March 2018.

The inclusion criteria were as follows: Patients who received nivolumab and had previously received platinum-containing chemotherapy for advanced NSCLC with bone metastases.

Patients were excluded if they had received immunotherapy or any additional concurrently administered antineoplastic therapies. Patients were also excluded from the analysis if they had previously undergone surgery, RT, or other local interventional therapies to the metastatic bone.

A total of 15 patients (11 men and 4 women) were included in this study (Table I). The median age was 67 years (range, 40-75 years) at the time of receiving nivolumab treatment. Thirteen patients had adenocarcinoma, and two patients had squamous cell carcinoma. Two patients had recurrent NSCLC after lung surgery with curative intent, and 13 patients had stage IV NSCLC at the time of presentation. All but one patient had metastases to organs other than bone (93\%). The median number of analyzed bone metastases was 2 (1-8). The locations of bone metastases were vertebral bone (20), ilium (6), rib (6), scapula (3), and others (6). The median follow-up time of this study was 12.2 months (1-23).

Treatment. Patients had received $3 \mathrm{mg} / \mathrm{kg}$ nivolumab as an intravenous infusion every 2 weeks until progressive disease (PD) or unacceptable toxicity was observed. Prior to nivolumab therapy, the median number of chemotherapy regimens was 2 (1-9) (Table II). All patients previously received platinum-based therapy, and 5 patients previously received an epidermal growth factor receptor-tyrosine kinase inhibitor. BMAs were administered to 12 patients. Denosumab was administered to 2 patients, and zoledronic acid was administered to 10 patients.

Assessment of study outcomes. All patients were assessed by CT (Aquilion, Canon) at $120 \mathrm{kV}$ and slice thickness of $5 \mathrm{~mm}$ from head to pelvis. Radiographic assessments were performed at the start of nivolumab therapy, and the first time after treatment at a median of 7 weeks (3-12 weeks). Two patients were assessed after 3 weeks for sudden aggravation of clinical symptoms; they were found to have disease progression on CT. Assessment by CT was done at a median of every 7 weeks (4-9 weeks) thereafter. Two authors (E.N., T.K.) evaluated the CT images, and any disagreements were resolved by consensus.

Tumor responses were defined as complete response (CR), partial response (PR), stable disease (SD), and PD according to RECIST1.1 (22). Responders were classified as either CR or PR, and non-responders were classified as either PD or SD.

For assessing bone lesions, all images were viewed with routine bone window settings (window level $200 \mathrm{HU}$, window width 2,000 HU). Patients were classified depending on their bone lesions at the start of nivolumab treatment as lytic ( $\mathrm{L}$ group) in 5 patients, mixed ( $\mathrm{M}$ group) in 2 patients, blastic (B group) in 1 patient, lytic and mixed (L/M group) in 1 patient, lytic and blastic (L/B group) in 2 patients, and mixed and blastic (M/B group) in 4 patients.

Radiological responses of bone metastases were assessed using the MDA criteria (Table III) (12-14). According to the 
Table I. Patient characteristics.

\begin{tabular}{lc}
\hline Characteristics & Total, $\mathrm{n}=15$ \\
\hline Sex & 11 \\
Male & 4 \\
Female & \\
Age, years & 67 (range, $40-75)$ \\
Median & \\
Histology & 13 \\
Adenocarcinoma & 2 \\
Squamous cell carcinoma & \\
Metastases & 8 \\
Lung & 7 \\
Liver & 3 \\
Brain & 1 \\
Adrenal gland & 5 \\
Pleural dissemination & 11 \\
Lymph node & 5 \\
Mutation of epidermal growth & \\
factor receptor & \\
\hline
\end{tabular}

MDA criteria by CT, CR is defined as complete fill-in or sclerosis of lytic lesions for lytic and mixed lesions or disappearance of the tumor signal for any lesion. PR is defined as development of a sclerotic rim around an initially lytic lesion or sclerosis of lesions previously undetected on CT, or partial fill-in or sclerosis of lytic lesions, regression of measurable lesion in any lesion, or a decrease in blastic lesions. PD is defined as an increase in size of any existing measurable lesions, the appearance of new lesions, or an increase in blastic/lytic lesions. SD is defined as no change in blastic/lytic lesions, no change of measurable lesions, or the appearance of no new lesions. A case of PR in one bone metastasis and SD in another bone metastasis was considered PR, and a case of PD in one bone metastasis and SD in another bone metastasis was considered PD. Responders were classified as either CR or PR, and non-responders were classified as either PD or SD.

Best overall response (BOR), overall response rate (ORR), disease control rate (DCR), time to response (TTR), and duration of response (DOR) were investigated by RECIST1.1 and the MDA criteria (bone metastases). BOR was defined as the best response designation recorded between the date of the first dose of nivolumab and the date of initial objectively documented tumor progression based on RECIST1.1 and the MDA criteria, or the date of subsequent therapy, whichever occurred first. ORR was calculated as the proportion of patients with a BOR of CR or PR. DCR was calculated as the proportion of patients with a BOR of CR or PR or SD. TTR was defined as the duration of time from first dose of nivolumab to the date of initial radiographic CR or PR. DOR was defined as the duration of response, defined as the time from first confirmed response to the date of initial radiographic progression for patients with CR or PR.

Progression-free survival (PFS) and overall survival (OS) were also investigated using the Kaplan-Meier method. PFS
Table II. Prior systemic therapy.

Prior chemotherapy

Total, $\mathrm{n}=15$

Number of prior chemotherapy

1

2

3

5

7

9

5

5

1

2

1

1

Type of prior chemotherapy

Platinum-based therapy

15

EGFR-TKI

5

EGFR-TKI, epidermal growth factor receptor-tyrosine kinase inhibitor.

was defined as the time from the first dose of nivolumab to the date of first documented progression of tumor and bone metastatic site based on RECIST1.1 and the MDA criteria or death from any cause. OS was defined as the time from the date of first dose to the date of death from any cause or last known date alive for patients who were alive at the time of data analysis.

Patients who neither progressed nor died were censored on the date of their last tumor assessment. Patients who died without reported previous progression were considered to have progressed on the date of their death. Patients who received subsequent cancer treatment without reported progression were censored at the date of the last tumor assessment before starting further treatment.

Statistical analysis. PFS (RECIST1.1 and MDA criteria) and OS were estimated using the Kaplan-Meier method. The prognostic significance of the following variables on survival was assessed: Age, sex, the number of bone metastases, the number of chemotherapy regimens prior to nivolumab therapy, BMAs, and bone lesions. Regression of bone metastases according to the MDA criteria was also assessed as a predictor of OS and PFS (RECIST1.1) in cases of lytic or mixed bone metastases (14 patients).

To assess risk factors for patients without disease control (PD) according to RECIST1.1 and non-responders (SD or PD) according to the MDA criteria, clinical data were assessed, including the following: Age, sex, the number of bone metastases, the number of chemotherapy regimens prior to nivolumab therapy, BMAs, and bone lesions. Risk factors for regression of bone metastases according to the MDA criteria were also assessed in patients without disease control (PD) according to RECIST1.1. The relation between tumor control (RECIST1.1) and bone response (MDA criteria) and TTS for both was also evaluated to investigate whether the MDA criteria can predict RECIST1.1.

For univariate analysis, the Mann-Whitney U-test was used to analyze continuous parameters, and Fisher's exact test was used for categorical parameters. Multivariate analysis was performed using multiple logistic regression analysis. 
Table III. MD Anderson criteria for evaluation of bone metastases.

\begin{tabular}{ll}
\hline Response type & \multicolumn{1}{c}{ Definition } \\
\hline Complete response & Complete fill-in or sclerosis of lytic lesions on CT \\
& Disappearance of tumor signal on CT \\
& Normalization of osteoblastic lesion on CT \\
& Sclerotic rim around initially lytic lesion or sclerosis of lesions previously undetected on CT \\
Partial response & Partial fill-in or sclerosis of lytic lesion on CT \\
& Regression of measurable lesion on CT \\
& Decrease in blastic lesion on CT \\
Progressive disease & Increase in size of any existing measurable lesions on CT \\
& New lesion on CT \\
Increase in blastic/lytic lesion on CT \\
No change in blastic/lytic lesion on CT \\
No change in measurable lesion on CT \\
No new lesion on CT
\end{tabular}

CT, computed tomography.

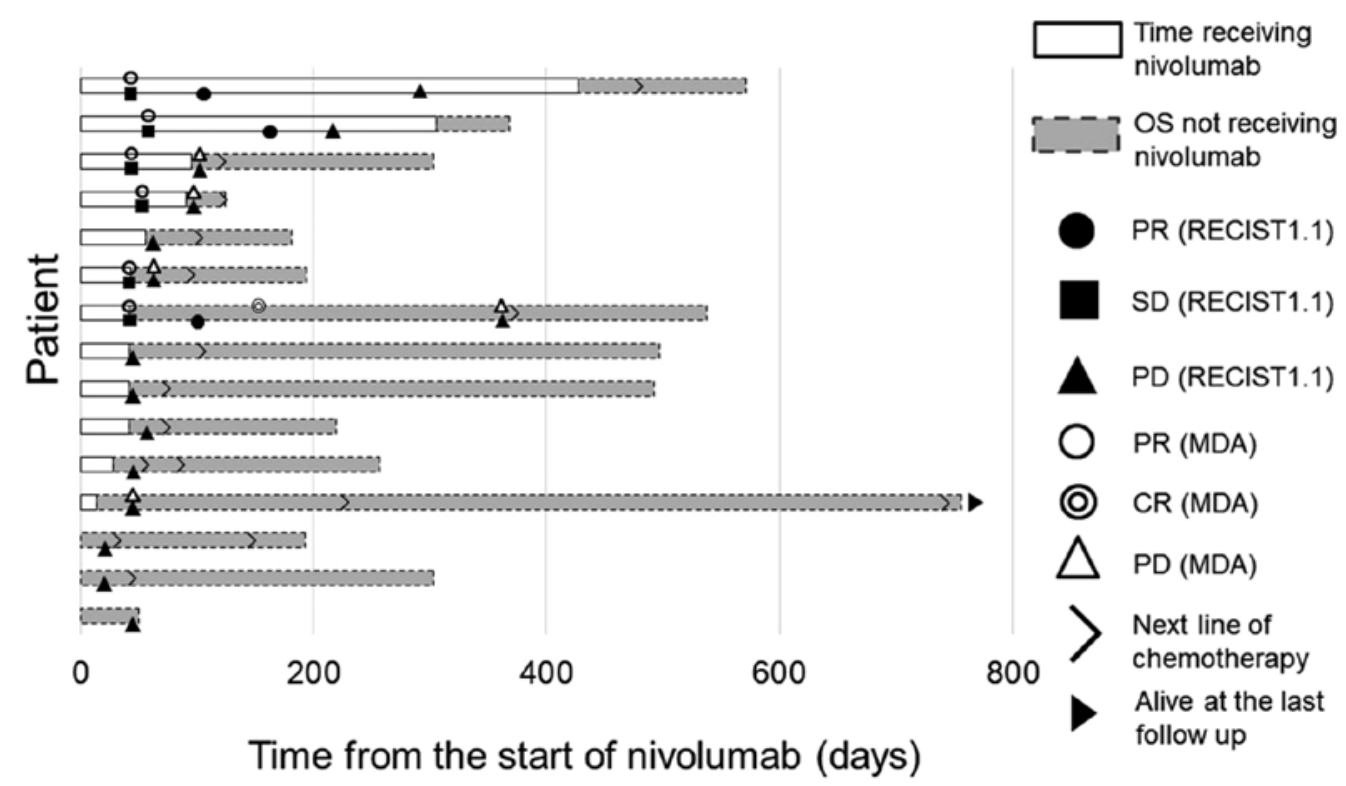

Figure 1. Swimmer plot. In RECIST1.1, BOR was PR in three patients (20\%), median TTR was 3.0 months (2.8-5.6 months) and median DOR was 5.8 months (1.5-9.3 months) as presented in the swimmer plot. In the MDA, BOR was CR in one patient and PR in five patients. Median TTR was 1.4 months (1.4-2.0 months) and median DOR was 6.2 months (0.7-17.7 months). RECIST1.1, Response Evaluation Criteria in Solid Tumors, version 1.1; BOR, best overall response; PR, partial response; TTR, time to response; DOR, duration of response; MDA, MD Anderson; CR, complete response; OS, overall survival; SD, stable disease; $\mathrm{PD}$, progressive disease.

For all analyses, associations were considered significant if the associated P-value was $<0.05$. All statistical analyses were performed with the statistical computing software $\mathrm{R}$ (R Version 3.5.0, R Core Team, Vienna, Austria).

\section{Results}

Nivolumab doses and treatment duration. A median of 4 doses of nivolumab was administered (1-23 doses), with a median treatment duration of 1.4 months (1-14 months). Fourteen patients discontinued nivolumab due to disease progression, and one patient discontinued due to adverse events (grade 2 diarrhea). All patients had PD during nivolumab treatment. Following the discontinuation of nivolumab, 13 (87\%) patients received subsequent chemotherapy; the median number of chemotherapy regimens was 1 (1-2). All but one patient had died by the time of the last follow-up.

Tumor control. With RECIST1.1, BOR was PR in 3 patients (20\%), SD in 3 patients (20\%), and PD in 9 patients $(60 \%)$. The ORR was $20 \%$, and the DCR was $40 \%$. Median TTR was 3.0 months (2.8-5.6 months), and median DOR was 5.8 months (1.5-9.3 months), as shown in a swimmer plot (Fig. 1). As reported in several studies $(5,6,23,24)$, the 
Table IV. Risk factors for tumor progression assessed by Response Evaluation Criteria in Solid Tumors, version 1.1 .

Number of patients

\begin{tabular}{|c|c|c|c|}
\hline Covariates & Patients with CR/PR/SD & Patients with PD & P-value \\
\hline \multicolumn{4}{|l|}{ Age, years } \\
\hline$<65$ & 3 & 3 & \\
\hline$\geq 65$ & 3 & 6 & 0.62 \\
\hline \multicolumn{4}{|l|}{ Sex } \\
\hline Male & 5 & 6 & \\
\hline Female & 1 & 3 & 0.60 \\
\hline \multicolumn{4}{|c|}{ Number of prior chemotherapy } \\
\hline$<3$ & 5 & 5 & \\
\hline$\geq 3$ & 1 & 4 & 0.58 \\
\hline \multicolumn{4}{|c|}{ Number of bone metastases } \\
\hline$<3$ & 6 & 4 & \\
\hline$\geq 3$ & 0 & 5 & 0.04 \\
\hline \multicolumn{4}{|c|}{ Bone modifying agent } \\
\hline Yes & 4 & 8 & \\
\hline No & 2 & 1 & 0.52 \\
\hline \multicolumn{4}{|l|}{ Bone lesion } \\
\hline Lytic only & 4 & 1 & \\
\hline Others & 2 & 8 & 0.09 \\
\hline \multicolumn{4}{|l|}{ MDA criteria } \\
\hline Responder & 6 & 0 & \\
\hline Non-responder & 0 & 9 & $<0.001$ \\
\hline
\end{tabular}

MDA, MD Anderson; CR, complete response; PR, partial response; SD, stable disease; PD, progressive disease.

long-term effect of nivolumab (durable response) was observed in one patient.

Univariate analysis showed that the number of bone metastases and the response according to the MDA criteria were the risk factors for patients without disease control (PD) according to RECIST1.1 (Table IV). Four of 10 patients (40\%) had PD among the patients with $<3$ bone metastases. On the other hand, all patients had PD among the patients with $\geq 3$ bone metastases. All responders according to the MDA criteria had a DCR of $100 \%$ with RECIST1.1, whereas the non-responders according to the MDA criteria had a DCR of $0 \%$ with RECIST1.1.

Median PFS was 1.9 months (0.4-12.1 months), with PFS of 33 and $20 \%$ at 3 and 6 months after the start of nivolumab treatment, respectively (Fig. 2). Univariate analysis showed that being a non-responder according to the MDA criteria was the only risk factor for PFS. PFS was 83 and $50 \%$ at 3 and 6 months after the start of nivolumab treatment, respectively, in patients who were responders according to the MDA criteria within 3 months. However, all patients who were non-responders according to the MDA criteria converted to PD within 3 months, which was significant $(\mathrm{P}<0.01)$ (Fig. 3).

Assessment of bone response by MDA criteria. Following nivolumab treatment, bone response was achieved in 8 lytic lesions in 6 patients (40\%). No change was seen in mixed and blastic lesions. There were 2 patients with PR in one bone lesion and SD in another bone lesion, which were considered PR. There was 1 patient with PD in one bone lesion and SD in another bone lesion, which was considered PD. In the remaining 12 patients, the same response was seen in all bone metastatic sites.

According to the MDA criteria, BOR was CR in 1 patient in group $\mathrm{L}$ and $\mathrm{PR}$ in 5 patients ( 3 patients in group $\mathrm{L}, 1$ patient in group L/M, and 1 patient in group L/B) (Table V). The ORR was $40 \%$. The median TTR was 1.4 months (1.4-2.0 months), which was the first-time assessment after the start of nivolumab therapy. The initial bone response was $\mathrm{OC}$ in 5 patients with a median TTR of 1.5 months (1.4-2.0 months) and regression of soft tissue extension in 1 patient with TTR of 1.4 months. Median DOR was 6.2 months (0.7-17.7 months), with 2 patients progression-free at the time of analysis. SD was achieved in 8 patients ( 1 patient in group L, 2 patients in group $M, 1$ patient in group B, 1 patient in group L/B, and 3 patients in group $\mathrm{M} / \mathrm{B}$ ). There was 1 patient with $\mathrm{PD}$ (one patient in group $\mathrm{M} / \mathrm{B}$ received RT for progression of an extraskeletal lesion of the right ilium, which was considered PD).

Univariate analysis showed that the number of bone metastases was the only risk factor for non-responders (Table VI). Four of 10 patients $(40 \%)$ were non-responders among the 


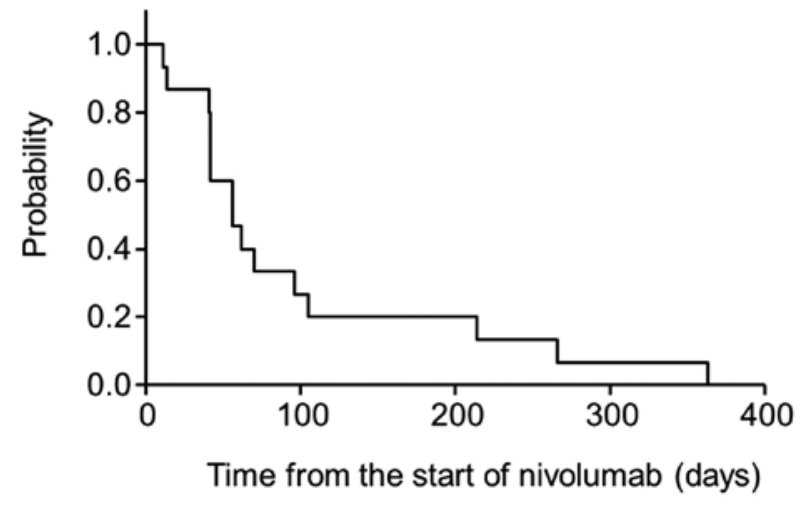

Figure 2. Progression-free survival (Response Evaluation Criteria in Solid Tumors, version 1.1). Median progression-free survival was 1.9 months (0.4-12.1 months), with progression-free survival of 33 and $20 \%$ at 3 and 6 months after the start of nivolumab therapy, respectively.

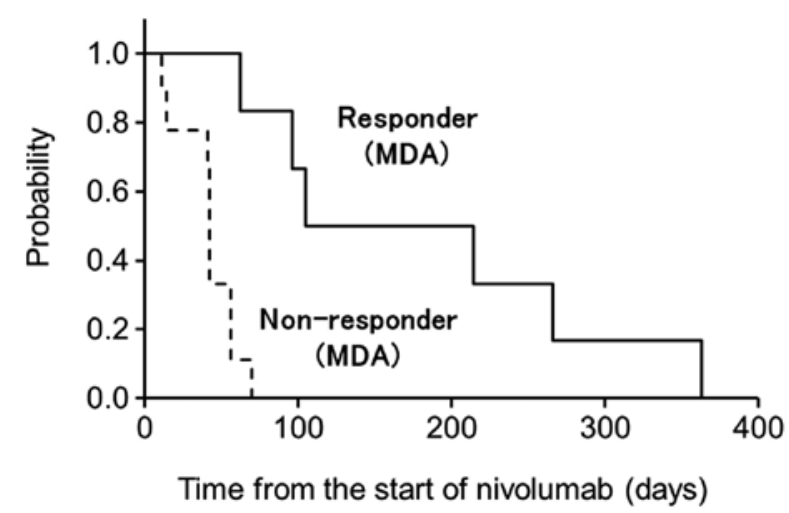

Figure 3. Progression-free survival (Response Evaluation Criteria in Solid Tumors, version 1.1) which depends on the MDA response criteria. Progression-free survival was 83 and $50 \%$ at 3 and 6 months after the start of nivolumab therapy, respectively, in patients who were responders according to the MDA criteria within 3 months. However, all patients that were non-responders according to the MDA criteria converted to progressive disease within 3 months, which was significant $(\mathrm{P}<0.01)$. MDA, MD Anderson.

patients with $<3$ bone metastases. On the other hand, all patients were non-responders among the patients with $\geq 3$ bone metastases.

Median PFS was 7.3 months (1.4-19 months), with PFS of 86 and $72 \%$ at 3 and 6 months after the start of nivolumab therapy, respectively (Fig. 4). Univariate analysis showed that no factors were correlated with PFS.

Relation between tumor control (RECIST1.1) and bone response (MDA criteria). Responders according to RECIST1.1 had significantly more responders according to the MDA criteria than non-responders according to RECIST1.1 $(\mathrm{P}<0.05)$. Although all responders (3 patients) according to RECIST1.1 were responders according to the MDA criteria (Table VII), in non-responders according to RECIST1.1, there were 3 responders and 9 non-responders according to the MDA criteria. In these 3 patients who were responders according to both RECIST1.1 and the MDA criteria, TTR occurred earlier according to the MDA criteria (1.4-2.0 months) than according to RECIST1.1 (2.8-3.0 months) in all patients.
Table V. Bone lesions and response according to the MD Anderson response criteria.

\begin{tabular}{lcccc}
\hline Bone lesions & CR & PR & SD & PD \\
\hline Lytic & 1 & 3 & 1 & \\
Mixed & & & 2 & \\
Blastic & & 1 & & \\
Lytic/Mixed & 1 & 1 & \\
Lytic/Blastic & & 3 & 1 \\
Mixed/Blastic & & & & \\
\hline
\end{tabular}

$\mathrm{CR}$, complete response; $\mathrm{PR}$, partial response; $\mathrm{SD}$, stable disease; $\mathrm{PD}$, progressive disease.

OS. At the time of analysis, 14 patients (93\%) had died. OS was 80 and $44 \%$ at 0.5 years and 1 year after the start of nivolumab therapy, respectively (Fig. 5). Univariate analysis showed that no factor was correlated with OS.

Case 1. A case of durable response of whole lesions and CR of bone metastases by nivolumab treatment is shown in Fig. 6 . The patient was 72 years old when he initially presented with lung adenocarcinoma and metastases to the lung, lymph node, and bone (lytic type bone lesion in third rib and T11 vertebra). He was initially treated with carboplatin, paclitaxel, and bevacizumab. Due to disease progression, he was switched to second-line therapy with nivolumab [original lesion (Fig. 6A), lung metastases (Fig. 6B) and left third rib metastases (Fig. 6C)].

After three doses of nivolumab (1.4 months), CT showed a stable appearance of the right original malignancy (Fig. 6D) along with decreases in size of the lung metastases and regression of soft tissue extension and $\mathrm{OC}$ of the left third rib metastases (Fig. 6E), compared with his baseline CT, which was considered SD according to RECIST1.1 and PR according to the MDA criteria. However, the nivolumab therapy was discontinued due to treatment-related grade 2 diarrhea, which could not be resolved with corticosteroid treatment. Although the patient received no further chemotherapy, each lesion showed decreases in size. Approximately 3 months after nivolumab treatment, decreases in size of the original malignancy (Fig. 6F) and lung metastases (Fig. 6G) were seen, which was considered PR according to RECIST1.1. Five months after nivolumab treatment, complete sclerotic fill-in of lytic lesions was demonstrated, which was considered $\mathrm{CR}$ according to the MDA criteria (Fig. 6H). Eight months after nivolumab treatment, a significant decrease in size of the original lesion (Fig. 6I), disappearance of the lung metastases (Fig. 6J), and further $\mathrm{OC}$ of the left third rib metastases (Fig. 6K) were noted. One year after nivolumab treatment, although these lesions were not changed, a new bone lesion was detected on bone scintigraphy, which was considered PD. The patient was subsequently treated with docetaxel and ramucirumab.

\section{Discussion}

The present study is the first to investigate the clinical results of nivolumab monotherapy in NSCLC patients with bone 
Table VI. Risk factors for non-responders of bone metastases assessed according to the MD Anderson response criteria.

Number of patients

Covariates

Patients with CR/PR

Patients with SD/PD

P-value

Age, years

$<65$

3

$\geq 65$

3

5

Male

6

Female

3

Number of prior chemotherapy

$<3$

5

$\geq 3$

Number of bone metastases

$<3$

$\geq 3$

Bone modifying agent

Yes

$4 \quad 8$

No

Bone lesion

Lytic only

Others

$\begin{array}{ll}4 & 1 \\ 2 & 8\end{array}$

1

8

$\mathrm{CR}$, complete response; $\mathrm{PR}$, partial response; $\mathrm{SD}$, stable disease; $\mathrm{PD}$, progressive disease.

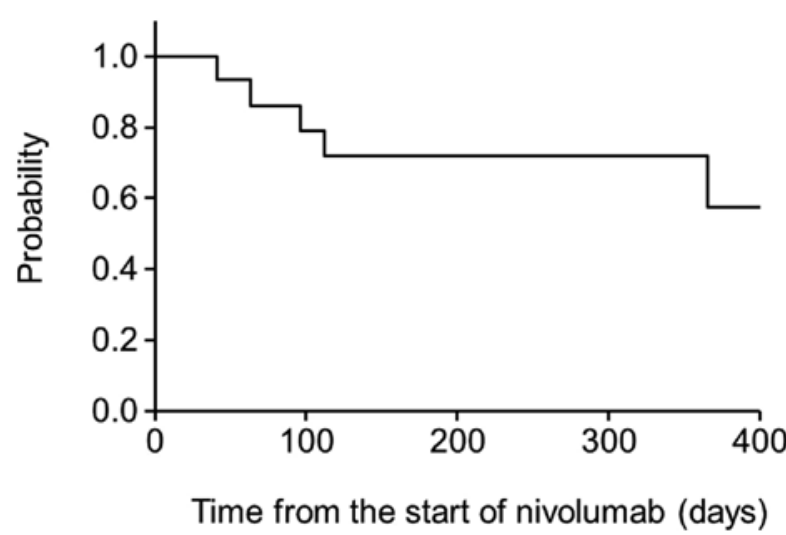

Figure 4. Progression-free survival (MD Anderson response criteria). Median progression-free survival was 7.3 months (1.4-19 months), with progression-free survival of 83 and $50 \%$ at 3 and 6 months after the start of nivolumab therapy, respectively.

metastases, and it found that nivolumab monotherapy resulted in OS of $44 \%$ at 1 -year post-treatment. The patient population was highly refractory, with almost one-third having received three or more systemic treatments previously. However, this result is consistent with previous studies reporting 1-year OS of $41-71 \%$.

In the present study, ORR according to RECIST1.1 was $20 \%$, which is consistent with previous studies reporting an ORR of $15-26 \%$. In numerous previous reports, molecular markers for prediction of response to nivolumab treatment were investigated, as the positive expression of PD-L1 and high tumor mutation burden were associated with significantly higher ORR than the others $(5,6,25,26)$. The present univariate analysis showed that the number of bone metastases and bone response assessed by the MDA criteria were the risk factors for patients with PD. Four of 10 patients (40\%) had PD among the patients with $<3$ bone metastases. On the other hand, all patients had PD among the patients with $\geq 3$ bone metastases. However, age, the number of chemotherapy regimens prior to nivolumab therapy, and the number of bone lesions were not significantly related. Bone is one of the most common sites affected by metastatic cancer $(1,2)$. The previous studies reported the existence of complex crosstalk among cancer cells, immune cells, and the bone microenvironment $(27,28)$. Although these molecular factors were not investigated, a greater number of bone metastases could indicate a more advanced stage of NSCLC, which would lead to a poor response.

In the present study, the median TTR of the cancer was 3.0 months, which is consistent with previous studies reporting TTR of 2.1-3.3 months (5-8). The median DOR was 5.8 months. Of note, as reported in several studies $(23,24)$, the long-term effect of nivolumab (durable response) was observed in some patients. Of the three patients who achieved an objective response, two (13\%) were progression-free for 9 and 12 months, respectively. Moreover, every lesion showed a decrease in size in one patient, although the patient received no further chemotherapy after the discontinuation of nivolumab, as shown in Fig. 6. 
Table VII. Relation of tumor control and bone response.

MDA criteria

\begin{tabular}{lcccc}
\cline { 2 - 5 } RECIST1.1 & CR & PR & SD & PD \\
\hline CR & & & & \\
PR & 1 & 2 & & \\
SD & & 3 & & \\
PD & & & 8 & 1 \\
\hline
\end{tabular}

RECIST1.1, Response Evaluation Criteria in Solid Tumors, version 1.1; CR, complete response; PR, partial response; SD, stable disease; PD, progressive disease; MDA, MD Anderson.

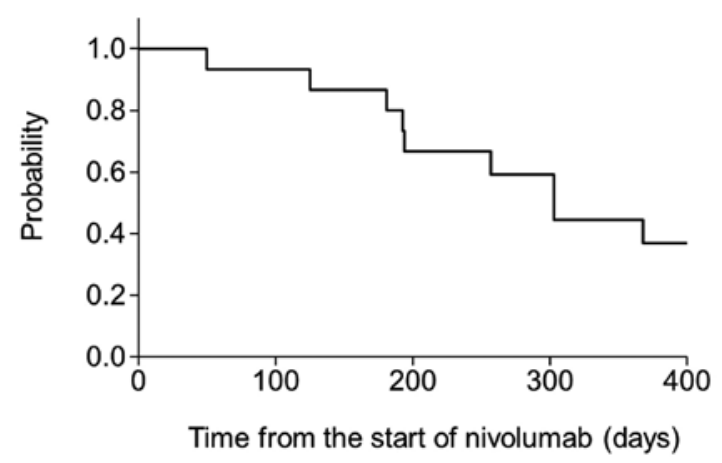

Figure 5. Overall survival. Overall survival was 72 and $57 \%$ at 0.5 and 1 year after the start of nivolumab therapy, respectively.

The present study is the first to investigate the effect of nivolumab therapy on metastatic bone lesions. Following nivolumab treatment, $\mathrm{OC}$ was noted in 8 lytic lesions in 6 patients on CT (40\%), of which partial sclerosis was seen in 5 patients (33\%), and complete sclerosis was seen in 1 patient (7\%). Four of 10 patients (40\%) were non-responders among the patients with $<3$ bone metastases. On the other hand, all patients were non-responders among the patients with $\geq 3$ bone metastases. A greater number of bone metastases could suggest a more advanced status of NSCLC, which would lead to the poor response.

Recently, several studies have indicated that $\mathrm{OC}$ is a marker of favorable response in various cancers, particularly in patients receiving chemotherapy and bone-targeted therapies (12-18). Some of these studies also showed that OC could be a marker of favorable response after chemotherapy and gefitinib in lung cancer $(15,16)$.

Rong et al reported a median interval of 2 months when OC occurred after chemotherapy in NSCLC patients with bone metastases (16). They showed that OC was a significant independent predictor of PFS on multivariate analysis, and patients with OC within 3 months had significantly higher 1-year PFS rates than patients with NOC $(\mathrm{P}<0.001)$. The authors also showed that patients in the OC group had a significantly higher 3-month DCR than those in the NOC group $(\mathrm{P}<0.001)$. The authors concluded that early $\mathrm{OC}$ within 3 months after the start of chemotherapy can predict the response to chemotherapy in NSCLC patients with bone metastases. Yamashita et al also reported OC as an indicator of good therapeutic response in lung cancer patients treated with gefitinib (15). Although all patients with OC were responders (RECIST1.1), only $40 \%$ were responders among the patients without $\mathrm{OC}$, which was significant $(\mathrm{P}<0.001)$. Moreover, there was a significant difference in the survival rate between the two groups $(\mathrm{P}<0.01)$, since the median survival of patients without OC was 170 days, while none of the patients with OC died within 170 days.

However, OC, as well as the regression of soft tissue extension, should be investigated in the assessment of bone metastases. Therefore, the MDA criteria were used in the present study. A significant relationwas demonstrated between the bone response and the tumor response in NSCLC patients with bone metastases who received nivolumab monotherapy. Response according to RECIST1.1 was significantly correlated with the response according to the MDA criteria $(\mathrm{P}<0.05)$. All responders (3 patients) according to RECIST1.1 were responders according to the MDA criteria. Importantly, the median time for bone response detected by CT was as early as 1.4 months (range 1.4-2 months). TTR according to the MDA criteria occurred earlier than that according to RECIST1.1 in all patients who were responders according to both RECIST1.1 and the MDA criteria.

Furthermore, bone response within 2 months was indicative of better PFS according to RECIST1.1 in NSCLC patients with bone metastases. In patients with bone response within 2 months, PFS was 83 and 50\% at 3 and 6 months after the start of nivolumab therapy, respectively. On the other hand, all patients were PD according to RECIST1.1 within 3 months were non-responder patients according to the MDA criteria.

Although early prediction of the effects of chemotherapy in NSCLC patients would help guide clinical practice, the optimal markers for predicting the outcomes of bone metastases in NSCLC patients have not yet been reported.

The present data suggest that, for patients who have received nivolumab monotherapy, being a responder according to the MDA criteria is indicative of better tumor responses than being a non-responder according to the MDA criteria, which could make predictions earlier than the previous research that assessed only OC in patients receiving chemotherapy (16) and gefitinib (15).

Moreover, these data could provide clinicians with a new means of evaluating the prognosis of NSCLC patients with bone metastases by monitoring bone metastases during nivolumab monotherapy. Considering that NSCLC with bone metastases is highly malignant, early prediction of the efficiency of chemotherapy should help improve patient outcomes. Thus, bone response assessed by the MDA criteria performed within 2 months of nivolumab monotherapy is useful for the early prediction of response and prognosis in NSCLC patients with bone metastases.

There were several limitations in this study. First, because this was a retrospective study, there could have been selection and verification biases; the patients had received various numbers of regimens of chemotherapy. Second, as we excluded patients from the analysis if they had previously undergone surgery, RT, or other local interventional therapies 
A

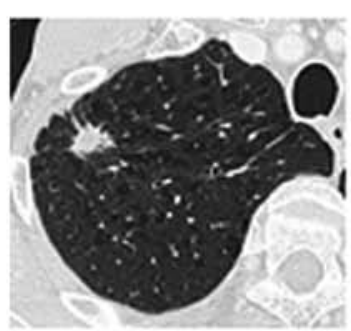

D

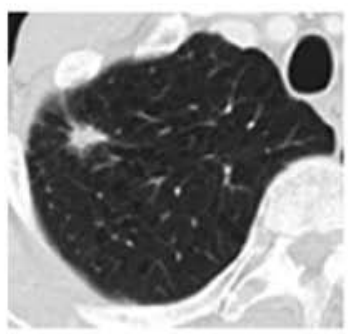

$\mathbf{F}$

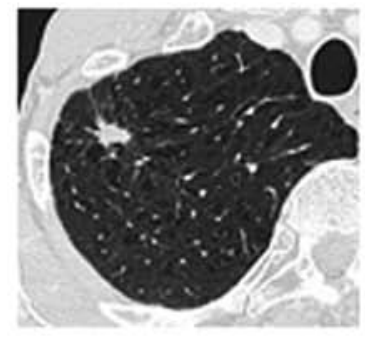

I

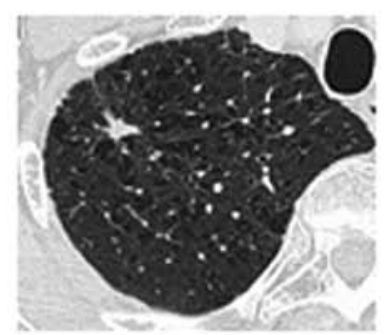

B

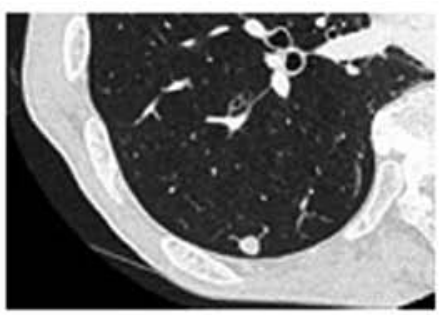

E

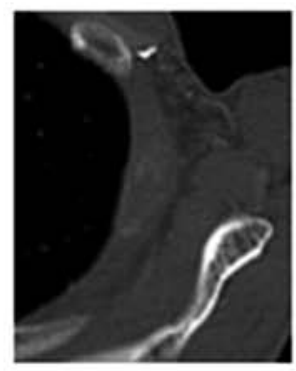

G

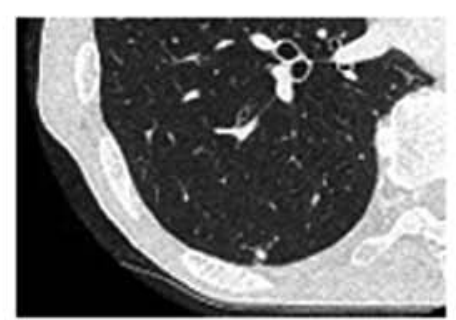

H

J

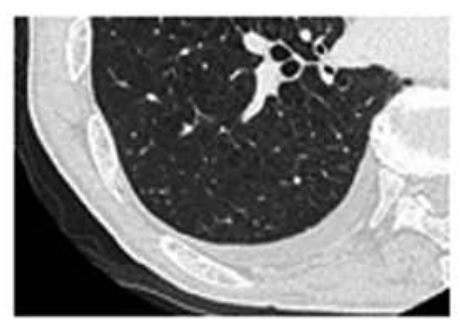

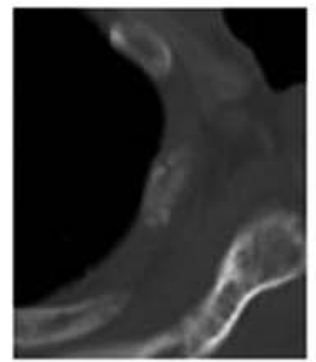

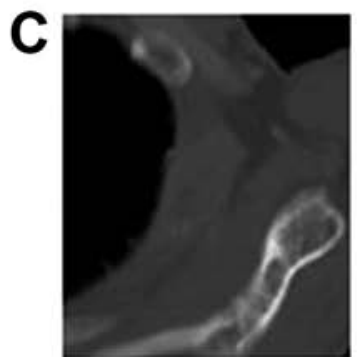

K

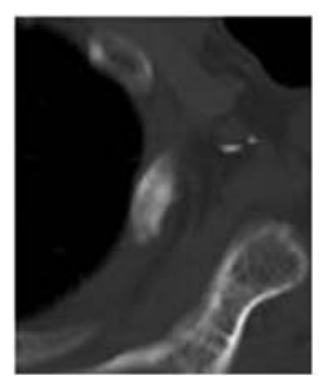

Figure 6. A case of durable response of whole lesions and CR of bone metastases by nivolumab treatment is shown. Due to disease progression, the patient was switched to second-line therapy with nivolumab. (A) Original lesion, (B) lung metastases and (C) left third rib metastases. After three doses of nivolumab (1.4 months), (D) CT showed a stable appearance of the right original malignancy along with (E) a decrease in the size of the lung metastases and regression of soft tissue extension and OC of the left third rib metastases compared with the patient's baseline CT, which was considered SD according to RECIST1.1 and PR according to the MDA criteria. At 3 months after nivolumab treatment, (F) decreases in size of the original malignancy and (G) lung metastases were observed, which was considered PR according to RECIST1.1. (H) At 5 months after nivolumab treatment, complete sclerotic fill-in of the lytic lesions was demonstrated, which was considered CR according to the MDA criteria. At 8 months after nivolumab treatment, (I) a significant decrease in size of the original lesion, $(\mathrm{J})$ disappearance of the lung metastases and $(\mathrm{K})$ further OC of the left third rib metastases were noted. CR, complete response; OC, osteosclerotic change; SD, stable disease; PR, partial response.

to the metastatic bone, the present study included a small sample size of only 15 patients. Third, median follow-up time of this study was 12.2 months, which was relatively short. These limitations were seen in other studies and is common in studies of NSCLC patients with bone metastases who have a relatively short survival $(15,16)$. A prospective study with a larger number of cases may be necessary to verify our hypothesis.

In conclusion, nivolumab monotherapy is effective for NSCLC patients with bone metastases. Bone response correlated significantly with tumor control. Early bone response to nivolumab monotherapy according to the MDA criteria can be useful for the early prediction of response and prognosis in NSCLC patients with bone metastases. Future studies with larger cohorts are needed to verify the results of the present study.

\section{Acknowledgements}

Not applicable.

\section{Funding}

No funding was received. 


\section{Availability of data and materials}

The datasets used and/or analyzed during the current study are available from the corresponding author on reasonable request.

\section{Authors' contributions}

EN, SS, TF and TT organized the study, and collected and analyzed data. RN, TKu and TO also analyzed data. YS, TKo, $\mathrm{NN}$ and DH treated the patients presented in this manuscript, and collected and analyzed data. All authors read and approved the final manuscript.

\section{Ethics approval and consent to participate}

All patients were informed about the effect and adverse events of nivolumab, the use of radiological examination, and other information, and patient consent was obtained. The present study was approved by the Ethics Review Board of Shikoku Cancer Center (approval no. 2017-26) and conducted in accordance with the World Medical Association Declaration of Helsinki.

\section{Patient consent for publication}

The patient described in the case report provided written informed consent for publication.

\section{Competing interests}

The authors declare that they have no competing interests.

\section{References}

1. Darvin P, Toor SM, Sasidharan Nair V and Elkord E: Immune checkpoint inhibitors: Recent progress and potential biomarkers. Exp Mol Med 50: 1-11, 2018.

2. Postow MA, Callahan MK and Wolchok JD: Immune checkpoint blockade in cancer therapy. J Clin Oncol 33: 1974-1982, 2015.

3. LaFleur MW, Muroyama Y, Drake CG and Sharpe AH: Inhibitors of the PD-1 pathway in tumor therapy. J Immunol 200: 375-383, 2018.

4. Zhang T, Xie J, Arai S, Wang L, Shi X, Shi N, Ma F, Chen S, Huang L, Yang L, et al: The efficacy and safety of anti-PD-1/PD-L1 antibodies for treatment of advanced or refractory cancers: A meta-analysis. Oncotarget 7: 73068-73079, 2016.

5. Hida T, Nishio M, Nogami N, Ohe Y, Nokihara H, Sakai H, Satouchi M, Nakagawa K, Takenoyama M, Isobe H, et al: Efficacy and safety of nivolumab in Japanese patients with advanced or recurrent squamous non-small cell lung cancer. Cancer Sci 108: 1000-1006, 2017.

6. Nishio M, Hida T, Atagi S, Sakai H, Nakagawa K, Takahashi T, Nogami N, Saka H, Takenoyama M, Maemondo M, et al: Multicentre phase II study of nivolumab in Japanese patients with advanced or recurrent non-squamous non-small cell lung cancer. ESMO Open 1: e000108, 2017.

7. Rizvi NA, Mazières J, Planchard D, Stinchcombe TE, Dy GK, Antonia SJ, Horn L, Lena H, Minenza E, Mennecier B, et al: Activity and safety of nivolumab, an anti-PD-1 immune checkpoint inhibitor, for patients with advanced, refractory squamous non-small-cell lung cancer (CheckMate 063): A phase 2, single-arm trial. Lancet Oncol 16: 257-265, 2015.

8. Borghaei H, Paz-Ares L, Horn L, Spigel DR, Steins M, Ready NE, Chow LQ, Vokes EE, Felip E, Holgado E, et al: Nivolumab versus docetaxel in advanced nonsquamous non-small-cell lung cancer. N Engl J Med 373: 1627-1639, 2015.

9. Brahmer J, Reckamp KL, Baas P, Crinò L, Eberhardt WE, Poddubskaya E, Antonia S, Pluzanski A, Vokes EE Holgado E, et al: Nivolumab versus docetaxel in advanced squamous-cell non-small-cell lung cancer. N Engl J Med 373: $123-135,2015$
10. Brodowicz T, O'Byrne $\mathrm{K}$ and Manegold $\mathrm{C}$ : Bone matters in lung cancer. Ann Oncol 23: 2215-2222, 2012.

11. Tsuya A, Kurata T, Tamura K and Fukuoka M: Skeletal metastases in non-small cell lung cancer: A retrospective study. Lung Cancer 57: 229-232, 2007.

12. Hamaoka T, Costelloe CM, Madewell JE, Liu P, Berry DA, Islam R, Theriault RL, Hortobagyi GN and Ueno NT: Tumour response interpretation with new tumour response criteria vs the World Health Organisation criteria in patients with bone-only metastatic breast cancer. Br J Cancer 102: 651-657, 2010.

13. Costelloe CM, Chuang HH, Madewell JE and Ueno NT: Cancer response criteria and bone metastases: RECIST 1.1, MDA and PERCIST. J Cancer 1: 80-92, 2010.

14. Hayashi N, Costelloe CM, Hamaoka T, Wei C, Niikura N, Theriault RL, Hortobagyi GN, Madewell JE and Ueno NT: A prospective study of bone tumor response assessment in metastatic breast cancer. Clin Breast Cancer 13: 24-30, 2013.

15. Yamashita Y, Aoki T, Hanagiri T, Yoshii C, Mukae H, Uramoto H and Korogi Y: Osteosclerotic lesions in patients treated with gefitinib for lung adenocarcinomas: A sign of favorable therapeutic response. Skeletal Radiol 41: 409-414, 2012.

16. Rong D, Mao Y, Yang Q, Xu S, Zhao Q and Zhang R: Early osteosclerotic changes predict chemotherapy response in non-small-cell lung cancer patients with bone metastases. Eur Radiol 28: 4362-4369, 2018.

17. Chang CY, Simeone FJ, Torriani M and Bredella MA: Quantitative contrast-enhanced CT attenuation evaluation of osseous metastases following chemotherapy. Skeletal Radiol 46: 1385-1395, 2017.

18. Amir E, Whyne C, Freedman OC, Fralick M, Kumar R, Hardisty $\mathrm{M}$ and Clemons $\mathrm{M}$ : Radiological changes following second-line zoledronic acid treatment in breast cancer patients with bone metastases. Clin Exp Metastasis 26: 479-484, 2009.

19. Vassiliou V, Kalogeropoulou C, Christopoulos C, Solomou E, Leotsinides $\mathrm{M}$ and Kardamakis D: Combination ibandronate and radiotherapy for the treatment of bone metastases: Clinical evaluation and radiologic assessment. Int J Radiat Oncol Biol Phys 67: 264-272, 2007.

20. Quattrocchi CC, Piciucchi S, Sammarra M, Santini D, Vincenzi B, Tonini G, Grasso RF and Zobel BB: Bone metastases in breast cancer: Higher prevalence of osteosclerotic lesions. Radiol Med 112: 1049-1059, 2007 (In Italian).

21. Nakata E, Sugihara S, Kataoka M, Yamashita N, Furumatsu T, Takigawa T, Tetsunaga T and Ozaki T: Early response assessment of re-ossification after palliative conventional radiotherapy for vertebral bone metastases. J Orthop Sci 24: 332-336, 2019.

22. Eisenhauer EA, Therasse P, Bogaerts J, Schwartz LH, Sargent D, Ford R, Dancey J, Arbuck S, Gwyther S, Mooney M, et al: New response evaluation criteria in solid tumours: Revised RECIST guideline (version 1.1). Eur J Cancer 45: 228-247, 2009.

23. Gettinger S, Horn L, Jackman D, Spigel D, Antonia S, Hellmann M, Powderly J, Heist R, Sequist LV, Smith DC, et al: Five-year follow-up of nivolumab in previously treated advanced non-small-cell lung cancer: Results from the CA209-003 study. J Clin Oncol 36: 1675-1684, 2018.

24. Nadal E, Massuti B, Dómine M, García-Campelo R, Cobo M and Felip E: Immunotherapy with checkpoint inhibitors in non-small cell lung cancer: Insights from long-term survivors. Cancer Immunol Immunother 68: 341-352, 2019.

25. Blons H, Garinet S, Laurent-Puig P and Oudart JB: Molecular markers and prediction of response to immunotherapy in non-small cell lung cancer, an update. J Thorac Dis 11 (Suppl 1): S25-S36, 2019.

26. Prelaj A, Tay R, Ferrara R, Chaput N, Besse B and Califano R: Predictive biomarkers of response for immune checkpoint inhibitors in non-small-cell lung cancer. Eur J Cancer 106: 144-159, 2019.

27. Yoneda T and Hiraga T: Crosstalk between cancer cells and bone microenvironment in bone metastasis. Biochem Biophys Res Commun 328: 679-687, 2005.

28. Sterling JA, Edwards JR, Martin TJ and Mundy GR: Advances in the biology of bone metastasis: How the skeleton affects tumor behavior. Bone 48: 6-15, 2011. 